\title{
Is Single Platelet Rich Plasma Injection Effective for Management of Long Head of Biceps tendinitis: A Case Control Study
}

\author{
Ashraf Elazab* and Ahmed Sonbol \\ Knee \& Shoulder and sport Traumatology center, Mansoura, Egypt \\ Mansoura university, faculty of medicine, Anesthesia, and pain management unit
}

Submission: January 22, 2021; Published: February 12, 2021

*Corresponding author: Ashraf Elazab, Knee \& Shoulder and Sport Traumatology Center, mahata square, Mansoura, Egypt

Abstract

Introduction: Multiple studies have reported that platelet rich plasma (PRP) injection is effective in multiple musculoskeletal conditions. This study aims to evaluate PRP local injection in the management of long head of biceps tendinopathy compared to xylocaine injection.

Methods: A total of 52 cases diagnosed with biceps tendinitis not responding to conventional conservative management were included in this study. They were divided into two equal groups; PRP group (26 cases who underwent PRP injection), and controls ( 26 cases who underwent xylocaine injection). All cases were subjected to history taking, clinical examination, and radiological investigations. After that, ultrasound guided PRP injection was done for all cases. At follow up, visual analogue score (VAS) and Oxford Shoulder score were assessed.

Results: No statistically significant difference was detected between both groups regarding patient or disease characteristics. However, VAS (rest and activity) and Oxford shoulder score were significantly improved after PRP injection compared to controls.

Conclusion: Ultrasound-guided PRP single injection appears to be a safe and effective method for treatment of long head biceps tendinopathy weather isolated or associated with other inflammatory shoulder pathology not indicated for surgery.

Keywords: Biceps tendinitis; Platelet rich plasma; Musculoskeletal ultrasound Level of evidence: III (Case Control Study)

\section{Introduction}

Biceps tendonitis is inflammation of the long head of the bicep's tendon. Despite of incomplete understanding of the clinical and biomechanical aspects of tendinitis of the long head of biceps, most researchers would agree that its pathology results in significant anterior shoulder pain [1-3]. Treatment options include various conservative interventions, including local injections weather steroid or platelet rich plasma (PRP), or surgical procedures, such as tenotomy, tenodesis, or transfer $3 ; 4 ; 18$. The decision usually based upon many factors, including the patient's general condition, severity of the condition, duration of manifestations, associated shoulder pathologies, and expectations12. Multiple growth factors have been identified in PRP which are essential for the initiation of wound healing. These factors include vascular endothelial growth factor, plateletderived growth factor, along with epithelial growth factor [4-6]. These factors play a crucial role in tendon repair. Nevertheless, the exact mechanism of PRP in mediating that action is still a matter of debate 14. In vivo application of PRP in tendon injuries has led to angiogenesis and formation of fibrovascular callus, which positively affected the catabolic demarcation of injured tendon matrix [7]. In the existing literature, PRP injection has been proved to be a safe and efficacious method for tendinopathy control in various parts of human body 19. In this study, we tried to evaluate PRP local injection as one of the non-surgical modalities in management of long head of biceps tendinopathy, compared to xylocaine injection.

\section{Methods}

This is a prospective randomized study that was conducted during the period between August 2018 and February 2019 in Mansoura University pain clinic and Knee \& shoulder and sport traumatology center in Mansoura, Egypt. We included 
cases diagnosed clinically and radiologically with long head biceps tendinopathy weather isolated or combined with other inflammatory peri shoulder pathology (including subacromial bursitis, supraspinatus tendinopathy, and subscapularis tendinopathy). All patients showed no response to conservative management including NSAIDs and physical therapy [7-10].

At the beginning of the study, 262 cases were diagnosed to have biceps tendonitis, from which 16 cases had the primary type. As regard the remaining cases, there was an associated pathology with biceps tendonitis. To confirm that tendonitis is the main source of pain, we performed intraarticular injection of $2 \mathrm{ml}$ xylocaine in addition to $2 \mathrm{ml}$ bupivacaine, and response was monitored. If more than $50 \%$ improvement was reported, these cases were excluded as the primary pathology was more likely to be intraarticular (210 cases), while if less than 50\% improvement was experienced (36 cases), the pathology was likely to be extraarticular (from the tendon itself) 13. Thus, a total of 52 cases were recruited for the current study, from whom 16 cases had primary biceps tendonitis, and the remaining cases had the secondary type $[11,12]$.

An informed written consent was obtained from all the included cases before complete explanation of the benefits and drawbacks of both injection procedures. Additionally, the study was approved by the local ethical committee of Mansoura University [13-16]. Diagnosis was obtained by clinical examination by shoulder surgeon (the first author) and re-evaluated by pain management specialist. Point tenderness over the bicipital groove was detected in all cases which rotate laterally and medially, with external and internal rotation of the shoulder, respectively, which can be differentiated from painful superficial structures, like the anterior deltoid, which do not move with arm rotation. A comparison between the affected and the other non-affected shoulder was usually done in our routine examination. Radiological evaluation was used for confirmation of clinical findings by ultrasound or MRI studies. Our cases were randomized into two equal groups; PRP group included 26 cases that underwent PRP injection, and control group which included the remaining 26 cases that underwent xylocaine injection.

\section{PRP Preparation}

We used the standard method for PRP preparation as described by Barker et al. 1, and type 1B PRP (leucocyte rich, no activation, platelet concentration $<5 \times$ serum) was used for all cases based on the Sports Medicine PRP Classification system 15. First of all, $10 \mathrm{ml}$ of blood was withdrawn and kept in a CPDA (citrate phosphate dextrose adenine) blood tube. Centrifugation of the tube content was done at 2700 revolutions per minute for 7 minutes.

Approximately, $2 \mathrm{ml}$ of PRP was collected from the central 'buffy coat layer' in the centrifuged tube. Injection technique. The long head of biceps was identified using the ultrasound probe in the bicipital groove. In the PRP group, $2 \mathrm{ml}$ PRP was infiltrated without any associated local anesthesia, while $2 \mathrm{ml}$ xylocaine and normal saline $0.9 \%$ mixture (1:1 ratio) was infiltrated in the control group. All kinds of analgesia and anti-inflammatory medications were stopped few days before and after injection. Also, cold fomentations were recommended to relieve pain related to injection site. Additionally, patients were advised to avoid heavy activities with the affected arm for 6 weeks after injection.

\section{Data Collection}

Clinical data, VAS score and Oxford shoulder score was calculated and collected from patients in our centers by the $1^{\text {st }}$ author (Elazab A) and reevaluated by the $2^{\text {nd }}$ author (Sonbol A) before injection, one-week and four weeks post injection [17-20].

\section{Evaluation}

Oxford Shoulder Score was used to assess pain, range of motion, shoulder stability and function to give a maximum total score of 486. It was assessed at the previously mentioned intervals. Visual analogue scores (VAS) (0 to 10) for pain (VAS pain) at rest and during activities were also recorded5.Patient Satisfaction was tested for all patients by asking about the degree of his or her total shoulder improvement, another method for patient Satisfaction was used by asking the patient about re- injection if the symptoms reappear again.

\section{Sample Size Calculation}

Sample size was calculated using Power Analysis and Sample Size software program (PASS) version 15.0.5 for windows (2017) with the post-injection pain during rest as the primary outcome. Patients were allocated into two groups: Study (PRP) group and Control group. The null hypothesis was considered as the absence of difference between treatment modalities regarding the post-injection pain during rest. To the best of our knowledge, no previous studies were conducted comparing both modalities regarding the post-injection pain during rest. A sample size of 26 patients in each group is needed to achieve $80 \%$ power $(1-\beta$ or the probability of rejecting the null hypothesis when it is false) and detect an effect size of 0.7 (large effect size) in the proposed study using two-sided two-sample z-test with a significance level ( $\alpha$ or the probability of rejecting the null hypothesis when it is true) of $5 \%$.

\section{Statistical Analysis}

The collected data were analyzed using IBM's SPSS statistics (Statistical Package for the Social Sciences) for windows (version $25,2017)$. Normality of data distribution was tested by ShapiroWilk test. A 95\% confidence interval was used for all tests. P value $<0.05$ was considered statistically significant. Quantitative variables were expressed as mean and standard deviation, whereas categorical variables were expressed as frequency and percentage. For pair-wise comparison of data (within subjects), 
the follow-up values were compared to their corresponding basal value using related-samples Friedman's two-way analysis of variance by ranks with Bonferroni correction of $\mathrm{p}$ value for multiple comparisons [21,22].

\section{Results}

The mean age of the included cases was 42.15 and 40.45 years in PRP and control groups respectively. Males represented 46.2 and $57.5 \%$ of cases in both groups respectively, whereas the remaining cases were females. Regarding the occupation of the included cases, manual workers represented 30.8 and $53.8 \%$ of cases, while housewives constituted 38.5 and $15.34 \%$ of cases in the same groups respectively. Besides, the remaining cases were employees. History of sport activity was positive in 19.2 and $38.5 \%$ of cases in both groups respectively. Diabetes mellitus was present in 30.8 and $38.5 \%$ of cases in the study groups. Neither of the previously discussed variables was found to be significantly different between the two groups. Table 1 illustrates these data.

Table 1: Demographic characteristics, history of diabetes and sports, onset of symptoms and affected tendons in the study participants.

\begin{tabular}{|c|c|c|c|c|c|}
\hline & & Study group $(n=26)$ & Control group $(n=26)$ & $95 \% \mathrm{CI}$ & p \\
\hline \multicolumn{2}{|l|}{ Age } & $42.15 \pm 11.235$ & $40.54 \pm 10.432$ & $-4.42,7.65$ & 0.593 \\
\hline \multirow{2}{*}{ Gender } & Male & $46.2 \%(12)$ & $57.7 \%(15)$ & $-0.15,0.39$ & \\
\hline & Female & $53.8 \%(14)$ & $42.3 \%(11)$ & & 0.405 \\
\hline \multirow{3}{*}{ Occupation } & Housewife & $38.5 \%(10)$ & $15.4 \%(4)$ & & \\
\hline & Manual worker & $30.8 \%(8)$ & $53.8 \%(14)$ & & 0.122 \\
\hline & Employee & $30.8 \%(8)$ & $30.8 \%(8)$ & & \\
\hline History of DM & & $30.8 \%(8)$ & $38.5 \%(10)$ & $-0.18,0.34$ & 0.56 \\
\hline History of Sport & & $19.2 \%(5)$ & $38.5 \%(10)$ & $-0.05,0.43$ & 0.126 \\
\hline Supraspinatus tendinitis & & $38.5 \%(10)$ & $53.8 \%(14)$ & $-0.11,0.42$ & 0.266 \\
\hline Subacromial bursitis & & $38.5 \%(10)$ & $23.1 \%(6)$ & $-0.4,0.09$ & 0.229 \\
\hline Subscapularis tendinopathy & & $7.7 \%(2)$ & $15.4 \%(4)$ & $-0.1,0.25$ & 0.385 \\
\hline Onset of symptoms & & $2.67 \pm 0.489$ & $2.85 \pm 0.834$ & $-0.55,0.21$ & 0.366 \\
\hline
\end{tabular}

Although there was no significant difference between the two groups regarding basal VAS either during rest or activities, postinjection assessment revealed the superiority of PRP injection in relieving pain compared to controls throughout all follow up visits $(p<0.05)$. These data are illustrated at (Table 2) \& (Figure 1).
Regarding Oxford shoulder score, it was not significantly different between the two groups before intervention. Nevertheless, follow up scores showed significant improvement compared to controls. These data are illustrated at (Table 3) \& (Figure 2).

Table 2: Basal and follow-up values of VAS score (during rest and activity) at one, two and four weeks in the studied patients.

\begin{tabular}{|c|c|c|c|c|c|}
\hline & & Study group $(n=26)$ & Control group $(n=26)$ & $95 \% \mathrm{CI}$ & $\mathbf{p}$ \\
\hline \multirow{4}{*}{ Rest } & Basal & $3.77 \pm 0.765$ & $3.88 \pm 1.211$ & $-0.68,0.45$ & 0.683 \\
\hline & One week & $2.19 \pm 0.694$ & $3.38 \pm 1.061$ & $-1.69,-0.69$ & $<0.001$ \\
\hline & Two weeks & $0.35 \pm 0.485$ & $2.88 \pm 0.816$ & $-2.91,-2.16$ & $<0.001$ \\
\hline & Four weeks & $0.35 \pm 0.485$ & $2.62 \pm 0.752$ & $-2.62,-1.92$ & $<0.001$ \\
\hline \multirow{4}{*}{ Activity } & Basal & $7 \pm 0.748$ & $6.96 \pm 1.148$ & $-0.50,0.58$ & 0.887 \\
\hline & One week & $3.96 \pm 0.599$ & $5.96 \pm 0.958$ & $-2.45,-1.55$ & $<0.001$ \\
\hline & Two weeks & $1.08 \pm 0.484$ & $5.23 \pm 1.032$ & $-4.60,-3.70$ & $<0.001$ \\
\hline & Four weeks & $1.08 \pm 0.484$ & $4.42 \pm 0.945$ & $-3.76,-2.93$ & $<0.001$ \\
\hline
\end{tabular}

Table 3: Basal and follow-up values of Oxford score at one, two and four weeks in the studied patients.

\begin{tabular}{|l|c|c|c|c|}
\hline \multirow{2}{*}{ Oxford } & \multicolumn{2}{|c|}{ Study group (n= 26) } & Control group (n= 26) & 95\% CI \\
& Basal & $31.23 \pm 62$ & $31.08 \pm 6.393$ & $-3.30,3.61$ \\
& One week & $41.54 \pm 2.267$ & $34.38 \pm 7.055$ & $4.24,10.07$ \\
& Two weeks & $45.88 \pm 1.071$ & $37.96 \pm 8.037$ & $4.73,11.12$ \\
& Four weeks & $49.19 \pm 3.262$ & $39.69 \pm 7.974$ & $<.11,12.89$ \\
\hline
\end{tabular}




\section{Orthopedics and Rheumatology Open Access Journal (OROAJ)}

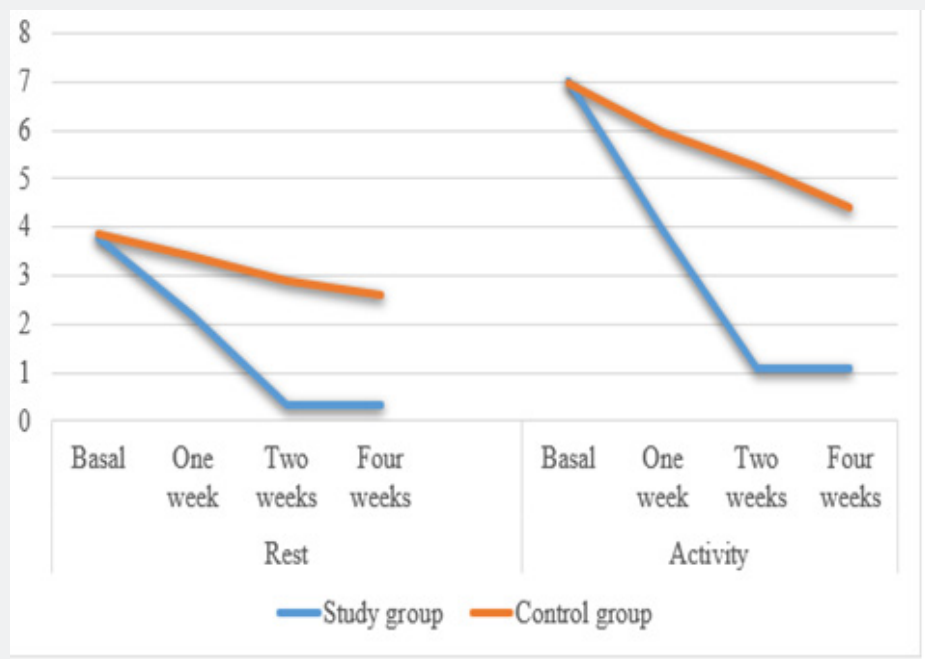

Figure 1: VAS changes in the study groups.

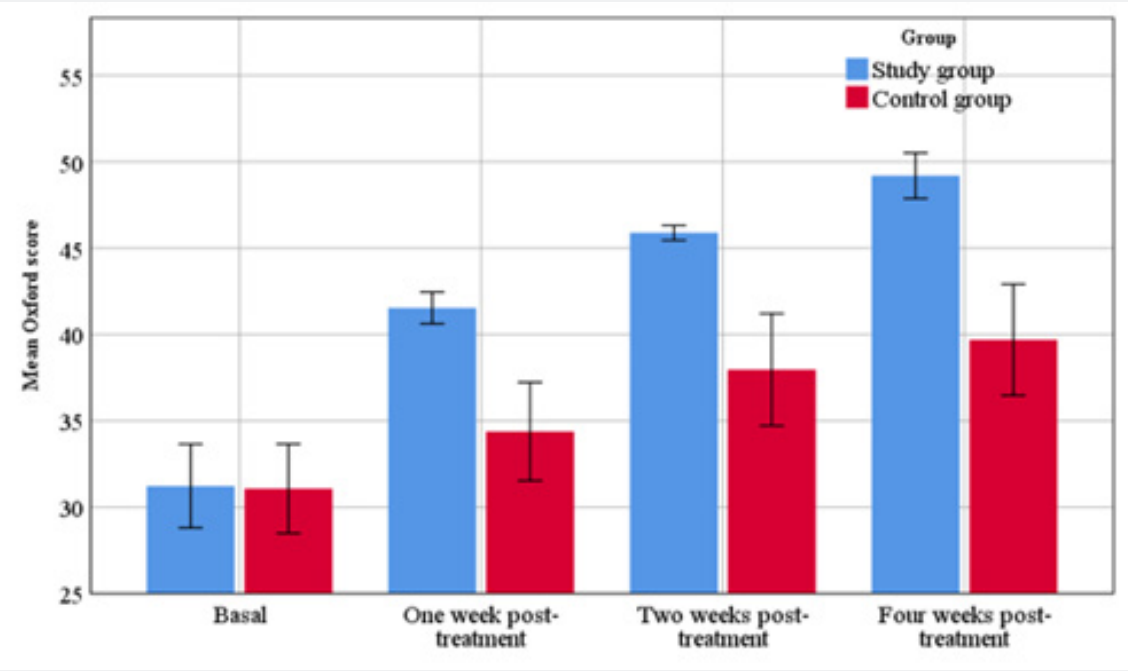

Figure 2: Oxford shoulder score changes in the study groups.

\section{Discussion}

This study was conducted at Mansoura University Hospitals and Knee \& shoulder and sport traumatology center in Mansoura aiming to evaluate PRP local injection as one of the non-surgical modalities in management of long head of biceps tendinopathy, compared to xylocaine injection. The included cases had a mean age of 42.15 and 40.45 years in PRP and controls, respectively. Increased activity is usually noticed in young age. So, there is more liability for tendinopathy due to excessive use either in males due to sports, or in females due to excessive domestic work. We used PRP alone without any additions as it was previously reported that addition of local anesthetics or steroids to PRP decreases its positive effect on tendon repair. Also, this addition may affect the viability of tendon cells at the injury site 2 .
Our results revealed that PRP was both effective and safe in managing such cases. Pain showed a significant decrease either during rest or activity in the PRP group compared to controls. Pain at rest decreased from 3.77 before intervention down to 2.19 , 0.35 and 0.35 at 1-,2-, and 4-week follow up visits, respectively. Also, VAS during activity decreased from 7 before injection down to $3.96,1.08$, and 1.08 at the same visits, respectively. Likewise, Oxford shoulder score showed a significant improvement as it increased from 31.23 before injection up to $41.45,45.88$, and 49.19 at the same follow up visits, respectively. All post-injection parameters were significantly better compared to controls ( $\mathrm{p}<$ 0.005). Nevertheless, controls showed temporary relief of pain and function because of its action as local anesthetic, not due to its effect on tendon repair as the previously mentioned study denied this effect [2]. 
In our study, we included cases with early stages of disease, which ensured excellent response with PRP injection, as it allowed tissue regeneration before the incidence of complete tissue fibrosis. PRP can rebalance the "healing" equation to move towards the formation of type I collagen instead of type III collagen that has inferior strength properties. Normally, type I collagen accounts for $65-80 \%$ of tendon collagen [9]. Also, PRP injection inside the tendon sheath led to significant improvement even in cases with secondary disease (having intraarticular lesions). Asthe tendon sheath is a natural extension of the joint cavity, it is plausible to assume that an injection into the sheath would result in intraarticular spread leading to improvement of intraarticular pathology as well [8].

The improvement noticed during rest was much better detected during activity. This could be explained by the fact that biceps tendon can maintain its compact form during rest as it is exposed to a little amount of stress. However, during activity, the tendon can undergo different stretch modalities leading to increased stress especially during arm flexion or supination [16].

Multiple reports have stated that platelet rich plasma (PRP) has an accelerating role in response to injury. Cellular response to an injurious agent is composed of four stages: hemostasis, inflammation, proliferation, as well as remodeling. Platelets are involved in the cellular or molecular processes during these four phases. Hemostasis is induced by plasma and platelets, whereas inflammation is mediated by leukocytes and activated platelets. Furthermore, tissue regeneration is influenced by growth factors released from platelet $\alpha$-granules. Accordingly, PRP leukocyte and growth factor content can influence inflammation and tissue regeneration respectively [17]. PRP composition and formulation affect the cellular environment at which it is present to mediate tissue inflammation and repair [20].

Also, PRP administration has multiple advantages; it could be easily prepared, and it is extracted from the patient own blood. Furthermore, no significant side effects are encountered like reported with steroids and non-steroidal anti-inflammatory drugs [21]. Sanli and his associates reported that there was a significant improvement in VAS during rest and activity with single injection of PRP for biceps tendonitis. However, that study was conducted for distal tendonitis cases. During rest, Vas decreased from 6 before intervention down to 0.5 at final follow up, whereas it decreased from 8 to 2 during movement at the same follow up ( $p$ $<0.002$ ). Moreover, elbow functional score showed a significant improvement as it increased from 63 before injection up to 90 at follow up $(\mathrm{p}<0.004)$ [18]. This study confirmed our findings regarding the efficacy of single PRP injection.

We preferred to perform single injection as it would provide multiple advantages including decreased financial cost, decreased hospital visits for injections, decreased the rate of anticipated complications, and thus, more patient satisfaction was noticed. Also, our study included biceps tendonitis cases, which would benefit from this single injection as tendon damage is not as severe as detected in rotator cuff tear or osteoarthritis cases. Therefore, more healing response should be anticipated with only single dose injection. Another study included 8 cases with bicipital tendinopathy following spinal cord injury. After PRP injection, there was a significant improvement of VAS score at 5 time points $(0,2,4,6$, and 8 weeks) for the injected arm. Also, no significant effects were reported after injection [11].

A recent Egyptian study conducted at Tanta University has reported that PRP was as effective as steroid injections in managing shoulder tendinopathies. VAS decreased from 8.3 before injection down to 2.3 at follow up ( $\mathrm{p}=0.0008)$. Also, Shoulder disability questionnaire showed a significant decrease from 90.3 down to 24.3 after the procedure. Furthermore, the range of motion was significantly improved in the PRP group $(\mathrm{p}<0.05) 10$. Another study has compared the local injection of PRP versus steroids in the management of biceps tendinopathy. PRP showed significantly higher efficacy compared to steroids. VAS decreased from 8 before intervention down to 1 six weeks after PRP injection, while it decreased from 9 to 8 in the steroid group. Besides, Disabilities of the Arm, Shoulder and Hand (DASH) score showed significant improvement in the PRP group (it decreased from 57.5 down to 9.3 after intervention), while it was worsened in the other group (from 58.3 up to 67.5 after steroid injection) [4].

Barker et al. included 6 cases diagnosed with biceps tendinopathy who underwent ultrasound guided PRP injection. Vas showed a significant improvement either during rest or activity (from 2.25 to 0 at rest, and from 7.25 to 1.3 during activity). Also, Mayo Elbow Performance Score significantly improved from 68.3 to 95 after injection 1 . This study has some limitations, first of all, the relatively small sample size. In addition, longer follow up period should have been recorded. Therefore, more studies should be conducted in the near future to cover thee missing perspectives.

\section{Conclusion}

Ultrasound-guided PRP single injection appears to be a safe and effective method for management of long head biceps tendinopathy weather isolated or associated with other mild to moderate inflammatory shoulder pathology not indicated for surgery.

\section{References}

1. Barker SL, Bell SN, Connell D, Coghlan JA (2015) Ultrasound-guided platelet-rich plasma injection for distal biceps tendinopathy. Shoulder \& elbow 7 : 110-114.

2. Carofino B, Chowaniec DM, Mc Carthy MB, Bradley JP, Delaronde S, (2012) et al. Corticosteroids and local anesthetics decrease positive effects of platelet-rich plasma: an in vitro study on human tendon cells. Arthroscopy: the journal of arthroscopic \& related surgery 28: 711719.

3. Churgay CA (2009) Diagnosis and treatment of biceps tendinitis and tendinosis. Am Fam Physician 
4. $80: 470-476$

5. Coria Serranía L, Villaseñor Moreno JC, Sánchez Ortíz ÁO, Herrera Flores R, (2017) Comparación de la inyección peritendinosa de plasma rico en plaquetas versus la inyección peritendinosa de esteroide en el tratamiento de la tendinopatía crónica de la porción larga del bíceps braquial: estudio clínico no aleatorizado. Revista Mexicana de Medicina Física y Rehabilitación 28: 42-48.

6. Crichton N (2001) Visual analogue scale (VAS). J Clin Nurs 10:706-706.

7. Dawson J, Rogers K, Fitzpatrick R, Carr A (2009) The Oxford shoulder score revisited. Archives of orthopaedic and trauma surgery 129: 119123.

8. De Mos $\mathrm{M}$, van der Windt $\mathrm{AE}$, Jahr $\mathrm{H}$, van Schie HT, Weinans $\mathrm{H}$, et al (2008) Can platelet-rich plasma enhance tendon repair? A cell culture study. Am J Sports Med 36: 1171-1178.

9. Gofeld M, Hurdle MF, Agur A (2019) Biceps tendon sheath injection: an anatomical conundrum. Pain Medicine 20:138-142.

10. Halpern BC, Chaudhury S, Rodeo SA (2012) The role of platelet-rich plasma in inducing musculoskeletal tissue healing. HSS J 8: 137-145.

11. Ibrahim DH, El-Gazzar NM, El-Saadany HM, El-Khouly RM (2019) Ultrasound-guided injection of platelet rich plasma versus corticosteroid for treatment of rotator cuff tendinopathy: Effect on shoulder pain, disability, range of motion and ultrasonographic findings. The Egyptian Rheumatologist 41:157-161.

12. Ibrahim V, Groah S, Libin A, Ljungberg I (2012) Use of platelet rich plasma for the treatment of bicipital tendinopathy in spinal cord injury: a pilot study. Topics in spinal cord injury rehabilitation 18: 77-78.

13. Karthik K, Elias D, Tavakkolizadeh A (2018) Management of Calcific
Tendinitis of the long head of biceps-Lessons learned. International Journal of Orthopedics 5: 985-988.

14. Krupp RJ, Kevern MA, Gaines MD, Kotara S, Singleton SB (2009) Long head of the biceps tendon pain: differential diagnosis and treatment. J Orthop Sports Phys Ther 39:55-70.

15. Marx RE (2004) Platelet-rich plasma: evidence to support its use Journal of oral and maxillofacial surgery 62(4): 489-496.

16. Mishra A, Harmon K, Woodall J, Vieira A (2012) Sports medicine applications of platelet rich plasma. Curr Pharm Biotechnol 13(7): 1185-1195.

17. Raney EB, Thankam FG, Dilisio MF, Agrawal DK (2017) Pain and the pathogenesis of biceps tendinopathy. Am J Transl Res 9:2668.

18. Rozman P, Bolta Z (2007) Use of platelet growth factors in treating wounds and soft-tissue injuries. Acta Dermatovenerol Alp Pannonica Adriat 16(4):156-165.

19. Sanli I, Morgan B, van Tilborg F, Funk L, Gosens T (2016) Single injection of platelet-rich plasma (PRP) for the treatment of refractory distal biceps tendonitis: long-term results of a prospective multicenter cohort study. Knee Surg Sports Traumatol Arthrosc 24(7): 2308-2312.

20. Townsend C, Von Rickenbach KJ, Bailowitz Z, Gellhorn AC (2020) PostProcedure Protocols Following Platelet-Rich Plasma Injections for Tendinopathy: A Systematic Review. PM R 12(9):904-915.

21. Wasterlain AS, Braun HJ, Dragoo JL (2016) Contents and Formulations of Platelet Rich Plasma. In: Maffulli N, editor. Platelet Rich Plasma in Musculoskeletal Practice: Springer p. 1-29.

22. Zhou Y, Wang JH (2016) PRP treatment efficacy for tendinopathy: a review of basic science studies. Biomed Res Int 2016:9103792.

\section{Your next submission with Juniper Publishers will reach you the below assets}

- Quality Editorial service

- Swift Peer Review

- Reprints availability

- E-prints Service

- Manuscript Podcast for convenient understanding

- Global attainment for your research

- Manuscript accessibility in different formats

( Pdf, E-pub, Full Text, Audio)

- Unceasing customer service

Track the below URL for one-step submission https://juniperpublishers.com/online-submission.php 